\title{
Fidelidad y traición: reflexiones a partir de un montaje de Samuel Beckett
}

\author{
Fidelity and Betrayal: Reflections on a Staging \\ of Samuel Beckett
}

\author{
Emoé De La PARRA \\ Facultad de Filosofía y Letras \\ Universidad Nacional Autónoma de México | México \\ Contacto: emoedelaparra@filos.unam.mx
}

\begin{abstract}
Resumen
¿Es posible que un movimiento tan corrosivo y evanescente como el trazado por Beckett haya podido devenir en canon? ¿Qué significa eso? ¿Es deseable que así lo sea? Si es un canon, ¿qué tipo de fidelidad solicita? ¿A qué paradigma o valor debe su potestad? ¿Qué constituye su traición y desde dónde es juzgada como tal? La posibilidad de que la transmutación en lenguaje escénico de un texto pueda o deba ser considerada como una traición al material original cobra, en la obra de Beckett, un interés particular en virtud de la enorme tensión que este tópico ha generado, tanto de carácter teórico como en el marco del quehacer teatral, a raíz del celo exacerbado de parte de los detentores de los correspondientes derechos. Mediante el análisis puntual de las peculiaridades estilísticas y las tesis filosóficas de Samuel Beckett se reflexiona sobre la legitimidad de dicho celo y se cuestiona su pertinencia contrastándolo, a manera de ejemplo, con un montaje particular del relato "Primer amor". Este artículo es una llamada de atención hacia las distorsiones que se generan al acatar irrestrictamente un canon estético. Es, también, un relato detallado de algunos de los procesos implicados en un montaje escénico.
\end{abstract}

Palabras clave: Beckett, canon, traición, realismo, puesta en escena, "Primer amor"

\begin{abstract}
Is it possible that a movement so corrosive and evanescent as the one contrived by Beckett could have become a canon? What does it mean? Is it something to be desired? If it is a canon, what sort of fidelity does it entreat? To which paradigm or value does it owe its strength? What constitutes its betrayal and from what perspective can it be considered as such? And as a necessary inference, to what extent can or should the translation of a dramatic text into a staging become a betrayal? The possibility that the transmutation to stage language of a text could or should be considered a betrayal of its original source has, in Beckett's work, a particular interest due to the great tension that this topic has brought about both in the theoretical and in the dramatic aspects because of the exaggerated zeal of the holders of its legal rights. Through the careful analysis of Beckett's stylistic uniqueness and his philosophical thesis, I ponder about the legitimacy of said zeal, and I question its relevance using the example of a particular staging of the short story "First Love". This paper tries to show the distortions that take place when following an aesthetic canon unrestrictedly. It is also a thorough description of some of the processes required in a staging.
\end{abstract}

Keywords: Beckett, canon, tradition, realism, staging, "First Love" 


\section{Introducción: el "mismaking"}

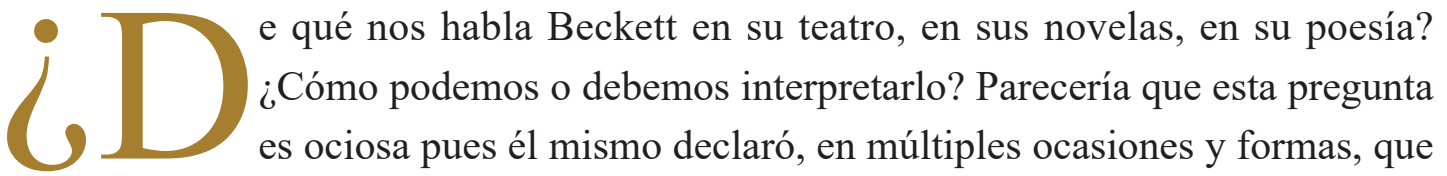
en sus obras no hay ningún misterio, dificultad o afirmación que deba ser esclarecida. Es más, reniega no sólo de la necesidad sino de la existencia misma, en palabras del propio Beckett, de ese "pequeño pájaro de la interpretación": "For in the forest of symbols (that aren't ones) the tiny birds of interpretation (which isn't) are never silent [Denn im Walde der Symbole, die keine sind, schweigen die Vöglein der Deutung, die keine ist, nie]" (De la Durantaye, 2016: 56).

La radicalidad de su posición nos desaconseja buscar respuestas donde ni siquiera hay enigmas. Sin embargo, aborda con meticulosa obsesión temas fundamentales de la condición humana. Así, por ejemplo, cuando Malone exclama: "La muerte debe tomarme por otro" (Beckett, 1997: 159), o "Todo está preparado. Excepto yo" (Beckett, 1997: 175), nos remite a esa velada certidumbre que todo hombre tiene sobre la muerte, a saber: que no le pertenece. Beckett vislumbra grandes verdades, pero en lugar de verterlas en una sentencia filosófica como hace Wittgenstein cuando escribe "Death is not an event in life. Death is not lived through" (Wittgenstein, 1996: §6.4311), o en un ensayo literario sobre la epifanía — a la manera de Proust—, nos ofrece metáforas y aseveraciones, tan rotundas como elusivas, que son más bien una invitación a la meditación y a un gozo estético inaugural.

En ello consiste su personal manera de hacer, o más bien de ese no hacer que él mismo llamó "la malfaçon créatrice voulue: willed creative mismaking" (De la Durantaye, 2016: 2). Con y sin su anuencia, el mecanismo de este extraño ejercicio y, en general la totalidad de su obra, ha ocasionado que se vierta mucha tinta en pos de la interpretación prohibida. No se necesita ser "particularly curious or particularly coarse to see a woman up to her midriff in a mound of earth, buried to the waist, in the waste, to ask what she is meant to mean" (De la Durantaye, 2016: 2). Semejante panorama inhibe cualquier respuesta porque las preguntas mismas que deberían contestar han caído en una misteriosa bancarrota. Sin embargo, tal vez tengamos el consuelo de dirigirlas, aun maltrechas como nos las entrega nuestro autor, a sus propios personajes.

\section{Los personajes}

Todos los personajes de Beckett aborrecen el vacío simbólico y por eso mismo están obligados a hablar, deben contar historias imparablemente para evitar ser tragados por 
"le silence dont l'univers est fait." (Beckett, 2012: 167). ${ }^{1}$ Podríamos pensar entonces que estos parlanchines contumaces pueden dialogar mejor que su autor con nosotros. Además, todos ellos son filósofos en la medida en que comparten con la voz del innombrable esa "estúpida obsesión por la profundidad" (Beckett, 1969: 52) que los impulsa a inquirir con tesón sobre el sentido de su vida y del universo; de esta suerte, al menos por oficio, son expertos del sentido. Tienen la ventaja suplementaria de que son eternos, pues a pesar de su vocación de suicidio se declaran incompetentes para el acto. Leemos en Fragmento de Teatro I: "No soy bastante desgraciado. Ésa ha sido siempre mi desgracia, desgraciado, pero no lo bastante" (Beckett, 2006: 315). Y si tantas virtudes no fueran suficientes, contamos con su probidad y bravura, pues estos "gladiadores moribundos" - Karl (2001) nos recuerda — "are probing the limits of a meaningless world and coming to grief because of their very integrity" (26) y nos dejan perplejos por su afanoso brío. Sucede, sin embargo, que nuestros potenciales interlocutores padecen de múltiples discapacidades. Están enfermos de culpa: "Desde el momento en que todavía es lo que se llama un ser vivo no ha lugar para equivocarse, es culpable" (Beckett, 1997: 136); y aquel afanoso delirio de buscar verdades — que no son tales - es la manifestación de una fuerza de la que nada saben y menos controlan: su lucidez es tan reveladora, como inútil para nuestros propósitos.

Así, mientras que a exégetas y pensadores Beckett les prohíbe enfáticamente ceder a la inclinación a buscar la solución del enigma primordial, no sólo porque éste yace fuera del mundo espacio-temporal (cf. Wittgenstein, 2018: $\S 6.342,6.372$ ), sino porque no se reconoce la existencia de enigma alguno, sus personajes, encadenados a un "impulso de vivir" carente de fundamento y motivos - idea heredada de Schopenhauer - no cesan de contar historias, pero no para mantenerse con vida al estilo Sherezade, sino porque no pueden hacer otra cosa. Y ahí los deja, peligrosamente asomados al despeñadero de los límites del mundo, desafiando la lógica de la razón que bien indica que, "Whereof one cannot speak, thereof one must be silent” (Wittgenstein, 1996: §7). A dichos personajes no podemos dirigirles pregunta alguna porque han perdido carnes e identidad para devenir voces, flujos de conciencia inasible. Pero, cabe preguntarse: ¿conciencia?, ¿de qué?

\section{¿Tradición o canon?}

La crítica en torno a Beckett parece indicar que posiblemente nos sea imposible renunciar a la búsqueda de un sentido, si no estrictamente lógico o racional, al menos como

\footnotetext{
1 En Beckett asistimos a la erosión paulatina e irreversible del poder de significación de las palabras que, al encarnarse en sus personajes, deviene catástrofe personal y rotunda.
} 
guía para interpretarlo correctamente. ¿Correctamente? Sí, he aquí la gran paradoja que anima la elaboración de esta reflexión. ¿Es posible acaso que la obra beckettiana, de vocación tan corrosiva y evanescente, devenga en canon?

Por supuesto que en el caso que nos ocupa la aplicación de dicho apelativo requiere de múltiples precisiones y quizá habría que adoptar el término de "serie" para referirse a la unidad que conforma, pues como bien señala De la Durantaye (2016):

Nor should it surprise that the finest study in French on Beckett, that of Bruno Clément, is titled L'œuvre sans qualités (The CEuvre without Qualities). If œuvre is a bit too masterful a name for ease of use in Beckett's case, and neither canon (despite Ruby Cohn's superb A Beckett Canon [2001] and Paul Auster's repeated nonironic use of "the Beckett canon" to discuss editorial decisions in the standard edition of Beckett's English works) nor corpus is likely to replace it, there is a term to which no objection can be made, if only because it was Beckett's own: the series. (109)

Pero allende a esta cuestión normativa, es un hecho que con afán de no desvirtuar el misterio, de cerrarle el paso a cualquier contrabando de "verdad", a cualquier dilucidación hermenéutica, en fin, a todo aquello que desvirtúe este "des-hacer" beckettiano o malverse el afán "de nada" de este personaje que no es filósofo, ni agnóstico, ni teólogo, ni iconoclasta, podría estarse gestando una especie de "canon" con visos casi míticos en cuyo nombre se ejerce una tutela que, independientemente de su potestad, podría tener efectos nocivos en la obra que pretende proteger. Ciertamente, es menester velar con celo el legado de Beckett para salvaguardar su alcance y profundidad, más aún cuando tiene un carácter tan elusivo, pero habría que cuidarse de no convertirlo en un credo que demanda observancia irrestricta y hasta devota. Si lo hiciéremos, se atemperaría el tono cáustico, el espíritu irreverente que elogió el propio Beckett en Joyce cuando, a la manera de Dante, le inyectó "vida nueva" a la anquilosada lengua inglesa (De la Durantaye: 2016). En rigor, estas cuestiones de valores, sean estéticas o de otra índole, son despreciadas también por sus personajes: "sachant que ces histoires de valeurs ne sont pas pour vous [...]" (Beckett, 2012: 61). Así pues, frente a la cristalización de esta tradición tenemos la posibilidad del anquilosamiento y otra faceta, legítima y necesaria, que a mi parecer remite a cuestiones formales como son el ritmo, las temáticas y los efectos. Dirimir esta cuestión, por más espinosa que sea, es de vital importancia y excede el objetivo de esta reflexión, pero creo que debe mantenerse abierta.

En el caso de las obras de teatro enfrentamos el obstáculo suplementario de la forzosa traducción del texto escrito en material escénico y, ya se sabe: toda traducción conlleva una posible traición. Todos los dramaturgos han tenido que lidiar con esta 
singularidad de los montajes en que siempre hay imprevistos que, revestidos de calamidad o fortuna, contaminan de realidad escénica la tersa literalidad de la obra dramática. En la actualidad suele distinguirse entre la obra escrita y un montaje particular de la misma, en virtud de que éste involucra elementos y fuerzas básicas de un proceso de composición peculiar y ajeno al que rige el texto: gestualidad visual, sonora y de movimiento, vestuario, musicalización, adaptación de época, etc.

A partir de la Comedia del Arte y de la tradición alemana representada por Bertolt Brecht, ${ }^{2}$ Heiner Müller y Botho Strauss, se ha establecido el nuevo concepto de "dramaturgista" (para distinguirlo del de "dramaturgo", que sigue estando asociado al autor de un texto de teatro). Esta perspectiva reconoce que la relación entre texto y puesta en escena subsiste, pero pone el énfasis en la singularidad de cada experiencia teatral y pugna por el reconocimiento categórico de su disimilitud. Este movimiento intenta revolucionar aquel acuerdo tradicional, que regía casi como un contrato, entre la pieza escrita y el modo preciso en que debería ser representada. Ahora se trata de construir cada vez, para cada texto dramático y en vista de cada uno de sus diversos montajes, ese acuerdo. Ciertamente, Beckett se habría colocado con rotundidad del lado de la tradición a este respecto, pero, en rigor, toda obra de teatro - y por ende las suyas“invocan” algún tipo de mediación (la dramaturgia de cada puesta). De esta suerte, se impone ponderar su postura a la luz de los nuevos paradigmas.

\section{Beckett y el lenguaje escénico}

Con esta perspectiva en mente, ¿cómo se lleva a escena una pieza de Beckett? Podría decirse que un montaje de Beckett se realiza, simple y llanamente, siguiendo las profusas indicaciones escénicas que dejó el autor, tanto por escrito como mediante videos elevados a la categoría de paradigmas. Pero al calificarlo así, al decir "simple y llanamente", se hace honor a pie juntillas al dictado - legítimo o no- de no buscar interpretaciones y sentidos ahí donde el "deshacer Beckettiano" las prohíbe tajantemente. Pero, ¿es deseable que esto se haga? Y, más importante aún, ¿es realmente posible hacerlo?

Creo que la clave para encontrar, si no una respuesta cabal a esta inquietud, por lo menos líneas de trabajo para la puesta en escena del material, requiere que desviemos la mirada de los lineamientos autorales y nos centremos, con oídos de metteur en scène, en lo que los personajes nos quieren indicar mediante lo que piensan y sienten. Esta

\footnotetext{
2 Brecht no sólo ejerció de "dramaturgista" sino que se rodeó de un amplo equipo de los mismos con tareas especializadas para cada montaje.
} 
propuesta, además, es una invitación hetedoroxa y atrevida a encarar al autor —en este caso a Beckett — con absoluto desenfado.

\section{Interrogar a los personajes}

Me centro en la primera de las dos sugerencias. Esta conduce hacia una manera peculiar de llevar a escena a los personajes, con mucha mayor independencia de lo que parecen admitir las acotaciones expresas del propio autor, y va a contracorriente de lo que se supone que es su estética sobre este punto.

Tanto actores como personajes, Beckett no se cansa de recordarnos, somos conciencias de... nada. Pero también somos, como Hamm declara, carne que no ha sido olvidada por la naturaleza y "i[...] respiramos, cambiamos! ¡Se nos cae el pelo, los dientes! ¡Nuestra lozanía! ¡Nuestro ideales!” (Beckett, 2006: 217). Y bien podemos seguir este camino para construir mentalidades que habiten estos seres de ficción y los conduzcan, llenos de vitalidad, exactamente por las vías férreamente trazadas del estilo de la obra y del espíritu beckettiano.

Para darle forma a esa "nada" debemos insuflarle vida: la nuestra, prestarle sobre todo nuestra carne. Pero he aquí que los Estragones, Vladimiros y, en fin, toda esa galería de moribundos, incluida la vehemente voz de No yo, sospechan que su existencia está abierta en carne viva, que es carne abierta en canal; pues, ¿de qué otra manera podríamos describir a estos "elders kept in garbage cans, figures buried to their midriffs in mud; [these] blind, maimed, mud-bespattered, wounded, and warped individuals" (De la Durantaye, 2016: 163). Ciertamente es una galería de moribundos pero hay que ver la cantidad de precisiones de imaginería realista con que están dotados sus mundos.

Estos sujetos sospechosos de su carne nos sobrecogen porque esa misma carne se asoma en sus parlamentos y heridas convertida en sospecha: sospechan que algo los domina y amenaza con devorarlos, sospechan que ese monstruo sin nombre - que en el fondo saben que es su propia conciencia - se sirve de sus propias palabras para acorralarlos en una jaula del tiempo, sospechan que los problemas titánicos que enfrentan son eventos incidentales trastocados por sus obsesiones y manías, sospechan que nada es inocente porque todo tiene origen en su propia culpa. Sospechan de muchas cosas, pero su suspicacia siempre derrama en aflicción y todas se materializan en tormentos muy precisos. A veces toman la forma de ritos y apegos que las ensucian de materialidad, como cuando Malone confiesa "el infecto sentimiento de piedad que tan a menudo he experimentado ante las cosas, sobre todo cosillas de madera o de piedra, y que me 
inspiraba el deseo de llevarlas conmigo y guardarlas siempre, de modo que las recogía y me las metía en el bolsillo, a menudo llorando" (Beckett, 1997: 117). ${ }^{3}$

Ciertamente, para los personajes de Beckett "speech is a chore, thought a joke, communication an impossibility, life a burden, and love long since cancelled" (De la Durantaye, 2016: 163). No obstante, a través de su conducta y de sus trayectorias en la trama algunas veces parecen burlar los designios del autor y apuestan por conjurar lo inefable y amueblar su mundo, sucumben a la necesidad de contar con un entorno tangible, poblado de cosas tan pedestres como una piedra que, sin dejar de fungir como tal piedra, asume también el destino de baluarte, de fortín, de refugio. Intermitente pero decididamente, los encontramos anclados a obsesiones, a ritos y apegos mediante los cuales conjuran, o al menos soslayan, la soledad y la incomunicación a las que Beckett pretende mantenerlos sujetos. Y por ello lloran por las piedritas, atesoran amuletos y deambulan en trayectorias que sugieren dibujos. ${ }^{4}$ En resumen, hacen trampa y se fugan del universo yermo en el que están confinados.

Otras veces el martirio se da en otro registro, incluso cuando son indudablemente pasivos. Muchos de ellos apenas si se mueven y parecen consumir toda su energía en cavilar sobre cuestiones intelectuales; sin embargo, es notable la pasión y la ferocidad con que se entregan a la tarea. Así, por ejemplo, Watt, uno de los grandes "filósofos" beckettianos, está menos concernido con sus pesquisas que con lo que puede hacer con ellas, le interesa menos constatar que "nada pasa" que la acuciante necesidad de hacer algo con esa nada (cf. De la Durantaye, 2016: 91).

Aunque poblada por sesudos metafísicos, la obra de Beckett está plagada de precisiones concretas. Es en esas precisiones de apariencia puramente circunstancial, en ese "monstruo" ubicuo que acosa secretamente a los personajes - posiblemente más concreto de lo que el Beckett pensador reconocería — donde debemos hurgar para encontrar los hilos que nos conduzcan a una estética del montaje, una estética que debe dar cuenta del realismo del que la obra está humanamente infestada.

\section{Beckett y el realismo}

Hablar de realismo para montar Beckett suena casi a herejía. El ingenio del "mismaking" lo tuvo entre sus más frecuentes víctimas: "the grotesque fallacy of a realistic art" (De la Durantaye, 2016: 24-25). Tampoco sus personajes parecen muy accesibles a un

\footnotetext{
3 En el montaje que se reseña más abajo se reproduce un fetiche similar mediante un juego de matatena con huesitos de durazno.

4 Objetos y acciones que conforman el material base para dar forma escénica a cualquier relato escrito.
} 
tratamiento realista cuando declaran lacónicamente: "Poco importa que haya nacido o no, que haya vivido o no, que esté muerto o sólo agonizante, haré lo que siempre he hecho, en la ignorancia de lo que hago, de quién soy, de dónde soy, de si soy" (Beckett, 1997: 81-82). Ante panorama tan desolador se antoja que lo único que nos queda por hacer — aparte de nada - es leer en voz alta y en tono neutral — refrenando toda tentación de "representar", "ilustrar" o cualquier otra cosa-, con todo rigor y distancia, las palabras exactas plasmadas en el texto.

Por añadidura, Beckett proscribió la búsqueda o asignación de cualquier tipo de coordenadas en el sentido literal (geográficas y temporales, coordenadas en el mundo) y ¡claro está!, con semejante restricción parece difícil incorporar un tratamiento realista. Empero, esta prohibición es olímpicamente ignorada por los personajes: ellos literalmente se mueren por averiguar dónde están y hacia dónde se dirigen. A propósito de esto De la Durantaye opina, y yo le concedo razón, que: "The result of depriving reader and spectator of concrete coordinates, of real-world locales in which to imaginatively set the story, is not, of course, the dismissal of the question asked at the outset of The Unnamable, 'Where now?' It is, on the contrary, to increase its urgency" (De la Durantaye, 2016: 99-100).

Esta rebeldía es signo fecundo para nuestros intentos si sabemos prestarle la atención adecuada y establecer con ellos lazos de complicidad, quizá a espaldas del mismo autor. Y, en efecto, mucho nos desvelan. Tal vez porque tanto ellos como nosotros padecemos la tiranía de ese acicate ciego, de esa "lucha por la vida" o "ímpetu vital" que obliga a Malone a levantarse porque "le quema el culo" (Beckett, 1997: 110), tal vez porque están constituidos de la misma carne que nosotros, estos parlanchines incontinentes nos franquean el paso hacia los entresijos de su funcionamiento y la forma de transmutarlos en materia escénica.

Así, por ejemplo, Malone nos da cátedra sobre "motivaciones", la materia prima por excelencia del oficio actoral: "Y cada uno tiene sus motivos, mientras se pregunta de vez en cuando lo que importan, y si justifican el ir allí adonde va en vez de a otro sitio, en vez de a ninguna parte" (Beckett, 1997: 90-91). Y más adelante añade: "Porque a la gente no le basta con sufrir, necesitan el calor y el frío, la lluvia y su contrario que es el buen tiempo, y además el amor, la amistad, la piel tostada y la insuficiencia sexual y gástrica por ejemplo, en resumen los furores y locuras demasiado numerosos afortunadamente para ser enumerados" (Beckett, 1997: 109).

En las obras podemos encontrar otras sugerencias veladas, relativamente ajenas a las anotaciones explícitas del escritor, de las que podemos valernos para construir la psicología de los personajes, con apego a los ritmos y formas que, en mi opinión, son el verdadero canon beckettiano. Pero para ello, y repito que quizá esta declaración es 
una especie de blasfemia, debemos abordar el montaje teatral de obras de esta estirpe desde una perspectiva realista.

\section{Una experiencia similar con Harold Pinter}

A una conclusión similar llegué en ocasión de la dirección de la puesta en escena de Viejos tiempos de Harold Pinter, en 1993. Sobre esta experiencia publiqué posteriormente el ensayo "El realismo de Harold Pinter en escena" (De la Parra, 1997). Ahí sostengo que la intención de Pinter era reflejar la realidad con la mayor fidelidad posible. Beckett, a pesar de de que rechaza expresamente el realismo, puede ser leído en la misma clave pues, como dice De la Durantaye (2016): "Beckett rejects realism not because it aspires to reflect the world around it, but because it so abjectly fails at the task" (25).

Para ilustrar lo anterior me remito a algunas consideraciones que expuse en el ensayo referido y que se aplican mutatis mutandis también a Beckett. Menciono sólo una de ellas. Dado que Pinter remite a una realidad definida por su inmanencia, para interpretar sus obras con vistas a traducirlas a un lenguaje escénico, encontré indispensable porfiar en el realismo pinteriano, es decir, escudriñar su apego, fiel y riguroso, a la lógica inmanente del material. Aventuré la idea de que la única manera de no restar fuerza a las metáforas del dramaturgo era construyendo la literalidad sobre la que se levantan y opté por asumir justamente la lógica realista de la que Pinter parece abjurar. Esa lógica consistió en construir personajes que no tuvieran consciencia de la trama sui generis en la que el autor los tiene enredados, que se comportaran como lo hace cualquier ser humano en una situación inteligible, que se les escapara por completo la visión y la misma posibilidad de adivinar el absurdo en el que están inmersos. Aposté a que la mejor manera de reproducir el impulso pinteriano consistía prima facie en perderlo. Intenté construir personajes resueltamente vivos, es decir, gobernados por el desconocimiento de sus verdaderas motivaciones, marcados por la ceguera de las paradojas que producen, signados por la ingenua convicción con la que defienden sus mundos incompatibles $y$, en particular, por la naturalidad y facilidad con la que se tragan, sin darse cuenta, tanto misterio. Concluí que para montar fielmente a Pinter, "para convertir su dramaturgia en material escénico, era necesario antes hacerla 'tratable', esto es: hablarle de 'tú', 'bajarle los humos', atemperar el vuelo filosófico y lírico, ahogarla en la materia viva del actor... domesticarla" (De la Parra, 1997: 198-202).

En lo que respecta a la traducción escénica de las obras de Beckett considero que debe aplicarse lo mismo. En muchas de sus obras las tramas y situaciones trazadas se acercan al absurdo y rayan en lo inteligible. Sin embargo, en lo que toca a la traducción escénica de dichas obras, debe buscarse construir personajes enteramente vivos, quiero 
decir, lo suficientemente vivos como para ser personajes teatrales. Y tal vez esa viveza se construye reproduciendo algo similar a nuestra propia ceguera, en respetar el hecho de que sólo esporádicamente vislumbramos las grandes verdades de nuestra condición y que, cuando lo hacemos, solemos estar guiados por nuestra intuición y protegidos por nuestra vehemencia. Por supuesto que en ningún momento debe perderse de vista el peligro de esta opción. Es muy frágil la línea que separa, por un lado, la minuciosa articulación de una desarticulación y, por otro la suplantación, la introducción contrabandeada de una articulación ajena, la reinstauración de una coherencia que traicionaría el estilo y el aliento de Beckett, pero del mismo modo que lo hice en la puesta escénica de Viejos tiempos de Pinter y en mi incursión como actriz en una obra de Beckett, creo que el riesgo no sólo vale la pena, sino que es insalvable.

\section{Caso paradigmático: un montaje de Primer amor}

Con ese mismo espíritu, que hace eco a la sugerencia expuesta arriba de encarar al autor con desenfado, en 1998 Antonio Algarra y yo, a la sazón constituidos en compañía teatral, nos dimos a la riesgosa empresa de llevar a escena el cuento "Primer amor" de Beckett. En la primera etapa contar con el entusiasta apoyo de José Sanchis Sinisterra, responsable de la traducción. En las temporadas que le siguieron y que se prolongaron intermitentemente de 1998 a 2006 la puesta tuvo un éxito considerable.

Recojo algunas ideas de lo que Sanchis Sinisterra escribió a propósito del montaje, porque alude al tema de las traducciones y traiciones. Describiendo como una gran osadía la empresa en la que nos embarcamos Antonio Algarra y yo, dice que se trata del último eslabón de una cadena de traiciones artísticas. Empieza por la traición que el propio Samuel Beckett llevó a cabo al tomar de Turgueniev el título de su relato, justamente porque le interesa apropiarse de las connotaciones idealistas y románticas que el autor original le impregnó. En segundo lugar, Sanchis Sinisterra confiesa que él también traicionó a Beckett en la medida en que convirtió un texto radicalmente literario en un libreto escénico.

Por último, el tándem Emoé-Algarra traicionó a quién esto escribe - con reticente tolerancia - al dar soporte femenino a un personaje no sólo ásperamente masculino, sino profundamente misógino, obligando a los espectadores a "borrar" la naturaleza real de la actriz para ver y escuchar en escena la tragicómica rememoración de un hombre que no supo compartir su soledad con una mujer. Pero no hay que inquietarse ante tal cúmulo de traiciones. Quizás la vida del arte radica precisamente en este juego infinito de infidelidades, de apropiaciones y expropiaciones, sobre todo cuando el motor que impulsa a los jugadores 
es como me consta en este montaje - la necesidad de confrontarse peligrosamente con el riesgo, de cuestionar la mismidad con la alteridad. (Sanchis Sinisterra, 1998: s. p.)

\section{Una mujer en el papel}

La "traición” primordial a la que se refirió Sanchis Sinisterra fue, como podrá imaginarse, el hecho de que la compañía haya optado porque una mujer diera vida a este personaje, misántropo declarado como otros surgidos del mismo crisol, pero con el agravante de una repugnante misoginia de la que hace alarde. En declaración expresa en los subsecuentes programas de mano, los responsables del montaje hicieron hincapié en que aquella decisión no representaba un manifiesto de índole alguna. Hace veinte años los temas en torno al género estaban lejos de ocupar la centralidad que hoy ocupan, pero, independientemente de su actualidad o pertinencia, no parecen haber estado en el horizonte de sus preocupaciones y objetivos. En sus declaraciones y en lo que se aprecia en la puesta misma, Algarra y yo tampoco nos ocupamos centralmente de la forma en que la problemática en torno al papel de la mujer y del feminismo está implícitamente presente en la obra beckettiana. Cabe añadir que, quizá porque el material se resiste a ello, poco se ha ocupado la academia del tratamiento de la mujer por parte de Beckett. ${ }^{5}$

En principio, en aquella puesta tampoco se apostó, o se jugó — sea esto válido o no- con la posibilidad del escándalo. Y en ello tuvieron franco éxito pues se dio el curioso fenómeno de que, a pesar de que no se disimulaba ni enfatizaba la trasposición de género, muchos espectadores se confesaron incapaces de pronunciarse sobre el género del figurante. Las razones para seguir este derrotero fueron resultado de una estudiada consideración de carácter estético. Los seres que pueblan esos universos, en rigor, son simplemente humanos. Ya lo dijo Frederick R. Karl (2001): "Not fortuitously, Beckett's characters are sexually vague" (33); y algo similar dijo Beckett por boca de Malone: "Quizá ponga al hombre y a la mujer en la misma historia, hay tan poca diferencia ente un hombre y una mujer, quiero decir entre los míos.” (Beckett, 1997: 10).

Efectivamente, en la puesta en escena referida se advierte el deseo de sugerir cierta indeterminación genérica, aquello que está — como muchos de estos seres dolidos que en las últimas obras de Beckett no alcanzan a tener cuerpo completo - en la antesala del ser. Tal y como lo manifestaron en diversas entrevistas los responsables del proyecto, su intención fue hacer visible, dotándolo de un cuerpo atroz, a un protohombre o

5 En términos generales, Beckett no abordó abiertamente cuestiones de género y destinó poco espacio a "la mujer". De manera sucinta, se puede decir que trata la sexualidad como fuente de irritación y de angustia, a partir del vínculo orgánico con otras preocupaciones como la vida y la muerte. 
protomujer que prácticamente se eclipsara para transparentar los horrores y suplicios de un alma que ni siquiera ha alcanzado la primera diferenciación humana. En cuanto a la manera de encarnar el personaje, según lo expresaron públicamente, consideraron la posibilidad de que el personaje fuera un feto, buscando así volver realidad palpable el pensamiento de Malone: "sí, he aquí que soy un viejo feto" (Beckett, 1997: 80). Y acorde con ello se especuló con presentarlo dentro de una probeta o convertirlo en un embrión dentro de una incubadora. La dura materialidad de la realidad (incluido el propio cuerpo de mujer del protagonista) los obligó, confesaron, a desistir de esta idea $\mathrm{y}$, cuando finalmente hubieron de descartarla, optaron por revestirlo de clochard en honor a algunas de las figuras de las primeras obras de Beckett. Empero, toda la puesta se ciñó al propósito de generar una especie de quimera, algo indefinible e inverosímil. Inverosímil, al parecer, también fue su atrevimiento.

\section{Conclusión}

Es necesario mantener abierta la discusión sobre el tópico que inspiró estas reflexiones, a saber, la necesidad de ponderar entre fidelidad y traición. Creo que la tradición de Beckett ha devenido en un canon cuyo funcionamiento y pertinencia ciertamente hay que repensar en virtud del carácter abiertamente esquivo, prácticamente prohibido, de las interpretaciones y reformulaciones de la obra, que la vuelve especialmente vulnerable a la malversación, por llamarla de alguna manera.

En contrapartida, la recelosa vigilancia con la que se dictamina la viabilidad de los montajes escénicos me parece que raya en un sofocamiento de su brío y puede resultar en un empobrecimiento lamentable. En última instancia, cabe preguntarse si el "willed creative mismaking" no escapa también al control de los legatarios de Beckett, y si el estilo irascible e irreverenete con el que libró todas sus batallas no es también parte de su legado. Creo que hay que aguzar el oído al escuchar lo que dice Beckett, y hago mía la sentencia de De la Durantaye: “The last words of Beckett's last drama, What Where are: 'Time passes. That is all. Make sense who may. I switch off.' Where Beckett's voices switch off is where ours switch on" (De la Durantaye, 2016: 133).

En lo que toca al montaje referido de Primer amor quiero señalar que, si bien fue un desacato a lo prescrito y cayó literalmente en lo proscrito, en mi opinión acató fielmente el espíritu del relato, tanto desde el punto de vista formal como por la eficacia con la que transmitió la temática de la obra, el "anti-mensaje" beckettiano. La mejor manera, si no es que la única, de persuadirlos de la justeza de mi opinión es invitarlos a ver — el teatro por definición está ahí para verse - las múltiples puestas de obras de Beckett que no se ciñen a pie juntillas a las indicaciones del autor. 


\section{Referencias bibliográficas}

Beckett, Samuel. (1969). El innombrable. Barcelona: Ed. Lumen.

Beckett, Samuel. (1997). Malone muere. Madrid: Alianza Editorial.

Beckett, Samuel. (2006). Teatro reunido. México, D.F: Tusquets Editores.

Beckett, Samuel. (2012). Molloy. París: Les Éditions de Minuit.

De la Durantaye, Leland. (2016). Beckett's Art of Mismaking. Cambridge: Harvard University Press.

De la Parra, Emoé. (1997). "El realismo de Harold Pinter en escena". Anuario de Letras Modernas, 7, 191-208. https://doi.org/10.22201/ffyl.01860526p.1996.7

KARL, Frederick K. (2001). "Waiting for Beckett: Quest and Request”. En Frederick K. Karl, A Reader's Guide to the Contemporary English Novel. Estados Unidos: Syracuse University Press. 19-39.

Sanchis Sinisterra, José. (1998). Nota al programa de mano de Primer amor. México, D.F.: Centro Cultural Helénico, CONACULTA.

Wittgenstein, Ludwig. (1996 [1922]). Tractatus Logico-Philosophicus: Hypertext of the Ogden Bilingual Edition. (C. K. Ogden, Trad.). Recuperado el 10 de enero de 2020 de https://www.kfs.org/jonathan/witt/tlph.html 